\title{
Effects of wall waviness and temperature variations on the fluid flow and natural convection in an inclined corrugated channel
}

\author{
Meysam Amini ${ }^{1 *}$, Mohammad Najafi ${ }^{2}$, Qaran Dorosti ${ }^{1}$, Hassan Biglarian ${ }^{1}$, Esmaeil Ghasemi ${ }^{1}$ \\ ${ }^{1}$ Energy Research Center, Research Institute of Petroleum Industry (RIPI), Tehran, Iran \\ ${ }^{2}$ Mechanical Engineering Department, Islamic Azad University, Science and Research Branch, Tehran, Iran \\ *Corresponding Author E-mail: meysamini@yahoo.com
}

Copyright $\odot 2015$ Meysam Amini et al. This is an open access article distributed under the Creative Commons Attribution License, which permits unrestricted use, distribution, and reproduction in any medium, provided the original work is properly cited.

\begin{abstract}
A parametric study involving the effects of some combinations of parameters, in particular, different combinations of the Rayleigh number, amplitude, temperature, and inclination angle of a two-dimensional long wavy-walled channel on a laminar incompressible fluid flow and natural convection within the channel is performed. The considered channel has an undulated wall as one side of the channel, and a parallel flat wall at a differentially different temperature as its counterpart. The channel sustains variable inclination angle, variable wavy wall amplitude, and variable temperaturedifference between its two walls. A perturbation technique in terms of the small waviness of the undulated wall is performed to obtain a set of non-linear ordinary differential equations for the main flow and its perturbations. Solving this set of equations determines the streamline and temperature profiles for the imposed varying parameters. The results reveal that the fluid velocity along the channel axis increases with increasing the Rayleigh number, but decreases with increasing the channel inclination angle. The results also show that eddies appeared due to the steep undulations intensify as the temperature-difference between the two channel walls increases. The veracity of the present work is demonstrated through comparing the obtained results with those available in the literature.
\end{abstract}

Keywords: Natural Convection; Inclined Channel; Wavy Wall; Perturbation Technique.

\section{Introduction}

The study of heat transfer from irregular surfaces, in particular, wavy configurations has become extensive, for their widespread applications during past years. Preventing thermal boundary layers formation and promoting capability of the fluid motion near the surface for corrugated and roughened geometries increase the heat transfer rates, thereby resulting in enhancement of heat transfer performance. Among the various applications of such heat transfer surfaces are wall undulation, plate heat exchangers, micro-electronic devices, design of solar collectors, transpiration cooling of re-entry vehicles and rocket boosters, film vaporization in combustion chambers, and cross-hatching on ablative surfaces.

Among early studies related to the subject of heat transfer from irregular surfaces, is the work of Lekoudis et al. [1] who analyzed the boundary-layer of compressible flows along a corrugated wall. Shankar and Sinha [2] solved the Rayleigh problem along a wavy surface using the perturbation technique. Lessen and Gangwani [3] investigated the effects of low magnitude wall undulations in order to capture the stability of the boundary layer in laminar form. Recently, Rees and Pop [4] studied the effects of stationary plane waves on the natural convection induced by a heated undulated surface in a porous medium numerically. All of the above studies were performed on a single infinitely long horizontal wavy wall configuration without considering the channel-flow argument.

The problem of buoyancy-driven flow in a long channel having a vertical corrugated wall and a parallel flat wall was for the first time solved analytically by Vajravelu and Sastri [5] using the perturbation method. Considering the presence of heat sources/sinks, they investigated the effects of the Prandtl numbers, frequency parameter, and wall- 
temperature ratio on the fluid flow and temperature fields. However, the work was conducted for very small Grashof numbers and no effects of the inclination angle and the wavy wall amplitude was considered. Later, Vajravelu [6] extended the method to analyze the fluid flow and heat transfer in horizontal corrugated channels. Their results for the main part of the solution were found to be in good agreements with those of the plane Poiseuille flow. Das and Ahmed [7] employed the perturbation technique to analyze the buoyancy-driven magnetohydrodynamic (MHD) flow and heat transfer for a viscous incompressible fluid confined between a long vertical undulated plate and a parallel flat plate. They considered the effects of relative temperature of the channel walls on the velocity and temperature profiles without considering the wavy wall amplitude and inclination angle effects. In a related study, Das and Deka [8] used a numerical approach to solve the buoyancy-driven heat transfer in the same domain as that studied in [7]. Patidar and Purohit [9] analyzed the buoyancy-driven convection of an incompressible fluid flow in a porous medium confined between two parallel vertical corrugated plates using the perturbation technique. The MHD convection flow in a vertical corrugated channel with temperature-dependent heat source was studied by Rao et al. [10] employing the perturbation technique. In another study, Vajravelu [11] conducted the perturbation method for the combined free and forced convection in hydromagnetic flows in vertical wavy channels with traveling thermal waves. He found that the stream function values become smaller in the presence of the magnetic field.

Recently, a few other analytical studies on the fluid flow and heat transfer in vertical channels having wavy walls have appeared in the literature. Choudhury and Das [12] developed an analytical solution based on the perturbation technique for the free convection flow in a vertical channel utilizing non-Newtonian fluid. They found that the skin friction at the wavy wall is an increasing function of the Prandtl and Grashof numbers. The effects of slip were investigated by Rajeev and Jain [13] in an analytical study of the MHD flow of a viscous incompressible fluid with a temperature-dependent heat source confined between a long vertical wavy surface and a parallel flat wall. They observed that the skin friction and the Nusselt number at the wavy wall are decreased with increasing the intensity of magnetic field. Guria and Jana [14] analyzed the two-dimensional mixed convection fluid flow and heat transfer in a vertical corrugated channel with travelling thermal waves embedded in a porous medium, and employed the perturbation technique. They investigated the effects of a number of non-dimensional parameters on the velocity and temperature profiles. However, no effects of amplitude of the wavy walls or variation of the channel walls temperature-dependent on the velocity and temperature distributions were reported.

Very recently, Muthuraj and Srinivas [15] studied the combined free and force convection considering both heat and mass transfer within a vertical corrugated porous channel with traveling thermal waves. Using the perturbation technique, the effects of different pertinent parameters, namely, the Hartmann number, Schmidt number, and the porosity parameter, on the flow fields and heat and mass transfer characteristics were explained. Umavathi and Shekar [16] investigated the combined convection fluid flow and heat transfer through a long vertical corrugated channel filled with porous material, employing linearization technique. They assumed long wave approximation for perturbation solution. Gireesha and Mahanthesh [17] conducted an analytical approach for combined heat and mass transfer of an unsteady magnetohydrodynamics viscoelastic fluid flow in an irregular vertical channel with coupled boundary condition using perturbation technique. They analyzed the effects of different pertinent parameters such as Sherwood number and Biot number on velocity and temperature fields. Kumar and Umavathi [18] conducted the perturbation technique to problem of steady two-dimensional natural convective flow in a porous medium between a long vertical undulated wall and parallel flat wall in the presence of a heat source utilizing a Walters fluid (model B'). They discussed the relevant flow and heat transfer characteristics, namely, skin friction and the rate of heat transfer at both walls, in detail. Umavathi and Shekar [19] conducted the same method to laminar mixed convection in a wavy-vertical channel filled with two unmixable viscous fluids. They found that the Grashof number, viscosity parameter, geometry ratio and conductivity ratio enhance the velocity component parallel to the flow direction.

A detailed review of the existing literature reveals that combined effects of the channel wavy wall amplitude (surface waviness), channel walls temperature-difference, and the channel inclination angle on the fluid flow and heat transfer characteristics within the wavy-walled channel have not yet been fully investigated. The present study, therefore, considers a buoyancy-driven fully developed fluid flow within an inclined corrugated channel. The effects of different combinations of a number of variable parameters including the inclination angle, the walls temperature-difference, the Rayleigh number, and the amplitude of the wavy wall on this channel-flow are investigated.

\section{Problem formulation}

Consider an inclined channel shown in Fig. 1. For the channel, the $\bar{x}$-axis is taken to be vertically upwards parallel to the flat wall, with the $\bar{y}$-axis being perpendicular to it. The channel can turn counter-clockwise with respect to point $O$ to change the channel inclination angle, $\varphi$, as shown in this figure. The wavy and the flat walls are represented by $\bar{y}=\bar{\varepsilon} \cos (\bar{L} \bar{x})$ and $\bar{y}=d$, respectively. The left and the right walls of the channel are maintained at differentially different temperatures of $\overline{T_{l}}$ and $\overline{T_{r}}$, respectively. The channel is erected in an incompressible medium at the ambient temperature of $\bar{T}_{\infty}$. The present study considers that segment of the channel within which the two facing wall growing boundary layers have already merged into a fully developed single buoyant stream rising through the column formed by the two walls. 


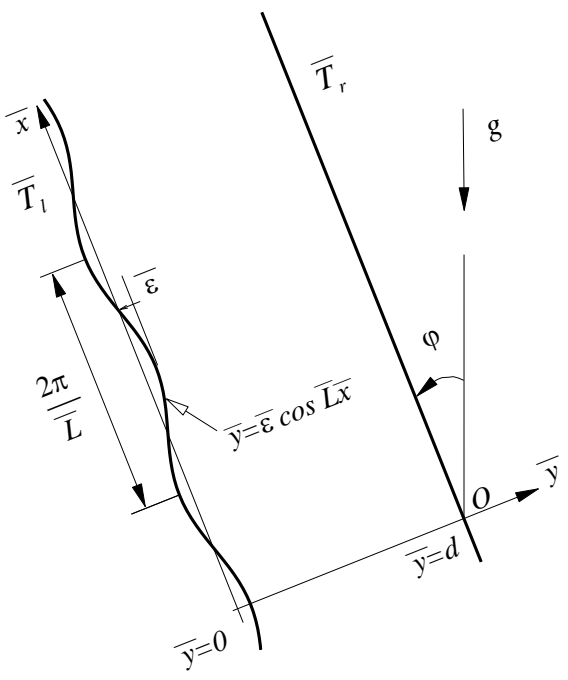

Fig. 1: Differentially-Heated Wavy Wall Channel Geometry

Depending on the value of $\bar{x}, \bar{L} \bar{x}$ represents a location on the wavy wall (Fig. 1). The amplitude of the wavy wall $\bar{\varepsilon}$ is assumed to be small enough for the perturbation technique to be applicable. The following analysis is conducted for $P r$ $=0.71$, and small wave numbers, i.e. small values of $\bar{L}$.

Equations (1), (2), (3), and (4) represent the continuity, $\bar{x}$ and $\bar{y}$ components of momentum, and energy equations for the steady, laminar, incompressible, and Newtonian fluid flow, respectively. The natural convection through the Boussinesq approximation is incorporated with the appropriate momentum equations, Eqs. (2) and (3).

$\frac{\partial \bar{u}}{\partial \bar{x}}+\frac{\partial \bar{v}}{\partial \bar{y}}=0$

$\left(\bar{u} \frac{\partial \bar{u}}{\partial \bar{x}}+\bar{v} \frac{\partial \bar{u}}{\partial \bar{y}}\right)=-\frac{1}{\rho} \frac{\partial \bar{p}}{\partial \bar{x}}+v\left(\frac{\partial^{2} \bar{u}}{\partial \bar{x}^{2}}+\frac{\partial^{2} \bar{u}}{\partial \bar{y}^{2}}\right)+g \beta\left(\bar{T}-\bar{T}_{\infty}\right) \cos \varphi$

$\left(\bar{u} \frac{\partial \bar{v}}{\partial \bar{x}}+\bar{v} \frac{\partial \bar{v}}{\partial \bar{y}}\right)=-\frac{1}{\rho} \frac{\partial \bar{p}}{\partial \bar{y}}+v\left(\frac{\partial^{2} \bar{v}}{\partial \bar{x}^{2}}+\frac{\partial^{2} \bar{v}}{\partial \bar{y}^{2}}\right)+g \beta\left(\bar{T}-\bar{T}_{\infty}\right) \sin \varphi$

$\rho c_{p}\left(\bar{u} \frac{\partial \bar{T}}{\partial \bar{x}}+\bar{v} \frac{\partial \bar{T}}{\partial \bar{y}}\right)=k\left(\frac{\partial^{2} \bar{T}}{\partial \bar{x}^{2}}+\frac{\partial^{2} \bar{T}}{\partial \bar{y}^{2}}\right)$

where $\bar{u}$ and $\bar{v}$ are the velocity components in the $\bar{x}$ and $\bar{y}$-directions, respectively. $\bar{T}$ Is the temperature, and $\beta$ is the thermal expansion coefficient of the fluid. The boundary conditions for Eqs. (1) - (4) are

$$
\begin{array}{llll}
\bar{u}=\bar{v}=0, & \bar{T}=\overline{T_{l}} \quad \text { on } & \bar{y}=\bar{\varepsilon} \cos (\bar{L} \bar{x}) \\
\bar{u}=\bar{v}=0, & \bar{T}=\bar{T}_{r} \quad \text { on } \quad \bar{y}=d
\end{array}
$$

In order to cast the governing equations and the boundary conditions into a dimensionless form, the following dimensionless variables are introduced

$$
\begin{array}{lll}
x=\bar{x} / d, & y=\bar{y} / d, \quad u=\overline{u d} / v, \quad v=\overline{v d} / v, \\
\varepsilon=\bar{\varepsilon} / d, & L=\bar{L} / d \quad, \quad \theta=\bar{T}-\overline{T_{\infty}} /\left(\overline{T_{l}}-\overline{T_{\infty}}\right), m=\left(\overline{T_{r}}-\overline{T_{\infty}}\right) /\left(\overline{T_{l}}-\overline{T_{\infty}}\right),
\end{array}
$$

where $m$ is the temperature scale, governing the variable temperature-difference between the two channel walls. Using the above dimensionless variables, the non-dimensional form of Eqs. (1) - (4) are written as 
$\frac{\partial u}{\partial x}+\frac{\partial v}{\partial y}=0$

$u \frac{\partial u}{\partial x}+v \frac{\partial u}{\partial y}=-\frac{\partial p}{\partial x}+\frac{\partial^{2} u}{\partial x^{2}}+\frac{\partial^{2} u}{\partial y^{2}}+G r \theta \cos \varphi$

$u \frac{\partial v}{\partial x}+v \frac{\partial v}{\partial y}=-\frac{\partial p}{\partial y}+\frac{\partial^{2} v}{\partial x^{2}}+\frac{\partial^{2} v}{\partial y^{2}}+\operatorname{Gr} \theta \sin \varphi$

$u \frac{\partial \theta}{\partial x}+v \frac{\partial \theta}{\partial y}=\frac{1}{\operatorname{Pr}}\left(\frac{\partial^{2} \theta}{\partial x^{2}}+\frac{\partial^{2} \theta}{\partial y^{2}}\right)$

where the Grashof number $G r$, the Prandtl number $P r$, and the Rayleigh number $R a$, are defined as

$G r=\frac{g \beta\left(\overline{T_{l}}-\bar{T}_{s}\right) d^{3}}{v^{2}}, \operatorname{Pr}=\frac{\mu c p}{k}$, and $R a=G r \operatorname{Pr}$

The dimensionless forms of the boundary conditions are

$$
\begin{array}{lll}
u=v=0 \quad, \quad \theta=1 \quad \text { on } & y=\varepsilon \cos (L x) \\
u=v=0 \quad, \quad \theta=m & \text { on } \quad y=1
\end{array}
$$

\section{Solution procedure}

To solve the governing equations by the perturbation technique, the field variables are written in the forms of expansions in powers of small parameter $\varepsilon$ [5]

$$
\begin{aligned}
& u(x, y)=u_{0}(y)+\varepsilon u_{1}(x, y)+\varepsilon^{2} u_{2}(x, y)+O\left(\varepsilon^{3}\right), \\
& v(x, y)=\varepsilon v_{1}(x, y)+\varepsilon^{2} v_{2}(x, y)+O\left(\varepsilon^{3}\right), \\
& \theta(x, y)=\theta_{0}(y)+\varepsilon \theta_{1}(x, y)+\varepsilon^{2} \theta_{2}(x, y)+O\left(\varepsilon^{3}\right), \\
& p(x, y)=p_{0}(x)+\varepsilon p_{1}(x, y)+\varepsilon^{2} p_{2}(x, y)+O\left(\varepsilon^{3}\right),
\end{aligned}
$$

where $u_{0}, \theta_{0}$, and $p_{0}$ are the mean parts, and $u_{k}, v_{k}, \theta_{k}$, and $p_{k}(k=1,2)$ are the perturbed parts. Substituting the above relations into Eqs. (7) - (10), and equating the coefficients of powers of $\varepsilon$ to zero yield the equations for the mean flow

$$
\begin{aligned}
& \frac{d^{2} u_{0}}{d y^{2}}+\operatorname{Gr} \theta_{0} \cos \varphi=0 \\
& \frac{d^{2} \theta_{0}}{d y^{2}}=0
\end{aligned}
$$

and the following equations for the perturbed flow

$$
\begin{aligned}
& \frac{\partial u_{1}}{\partial x}+\frac{\partial v_{1}}{\partial y}=0 \\
& u_{0} \frac{\partial u_{1}}{\partial x}+v_{1} \frac{\partial u_{0}}{\partial y}=-\frac{\partial p_{1}}{\partial x}+\frac{\partial^{2} u_{1}}{\partial x^{2}}+\frac{\partial^{2} u_{1}}{\partial y^{2}}+G r \theta_{1} \cos \varphi
\end{aligned}
$$


$u_{0} \frac{\partial v_{1}}{\partial x}=-\frac{\partial p_{1}}{\partial y}+\frac{\partial^{2} v_{1}}{\partial x^{2}}+\frac{\partial^{2} v_{1}}{\partial y^{2}}+G r \theta_{1} \sin \varphi$

$u_{0} \frac{\partial \theta_{1}}{\partial x}+v_{1} \frac{\partial \theta_{0}}{\partial y}=\frac{1}{\operatorname{Pr}}\left(\frac{\partial^{2} \theta_{1}}{\partial x^{2}}+\frac{\partial^{2} \theta_{1}}{\partial y^{2}}\right)$

$\frac{\partial u_{2}}{\partial x}+\frac{\partial v_{2}}{\partial y}=0$

$u_{0} \frac{\partial u_{2}}{\partial x}+u_{1} \frac{\partial u_{1}}{\partial x}+v_{1} \frac{\partial u_{1}}{\partial y}+v_{2} \frac{\partial u_{0}}{\partial y}=-\frac{\partial p_{2}}{\partial x}+\frac{\partial^{2} u_{2}}{\partial x^{2}}+\frac{\partial^{2} u_{2}}{\partial y^{2}}+G r \theta_{2} \cos \varphi$

$u_{0} \frac{\partial v_{2}}{\partial x}+u_{1} \frac{\partial v_{1}}{\partial x}+v_{1} \frac{\partial v_{1}}{\partial y}=-\frac{\partial p_{2}}{\partial y}+\frac{\partial^{2} v_{2}}{\partial x^{2}}+\frac{\partial^{2} v_{2}}{\partial y^{2}}+G r \theta_{2} \sin \varphi$

$u_{0} \frac{\partial \theta_{2}}{\partial x}+u_{1} \frac{\partial \theta_{1}}{\partial x}+v_{1} \frac{\partial \theta_{1}}{\partial y}+v_{2} \frac{\partial \theta_{0}}{\partial y}=\frac{1}{\operatorname{Pr}}\left(\frac{\partial^{2} \theta_{2}}{\partial x^{2}}+\frac{\partial^{2} \theta_{2}}{\partial y^{2}}\right)$

Following the similar procedure, the boundary conditions for the zeroth, first, and second-order equations are written as

$$
\begin{aligned}
& u_{0}=0, \quad \theta_{0}=1, \quad \text { on } \quad y=0 \\
& u_{0}=0, \quad \theta_{0}=m, \quad \text { on } \quad y=1 \\
& u_{1}=-\operatorname{Re}\left(e^{i L x} u_{0}^{\prime}\right), \quad v_{1}=0, \quad \theta_{1}=-\operatorname{Re}\left(e^{i L x} \theta_{0}^{\prime}\right), \quad \text { on } \quad y=0 \\
& u_{1}=0, \quad v_{1}=0, \quad \theta_{1}=0, \quad \text { on } \quad y=1
\end{aligned}
$$

$u_{2}=-\operatorname{Re}\left(e^{i L x} u_{1}^{\prime}\right), v_{2}=-\operatorname{Re}\left(e^{i L x} v_{1}^{\prime}\right), \theta_{2}=-\operatorname{Re}\left(e^{i L x} \theta_{1}^{\prime}\right), \quad$ on $\quad y=0$

$u_{2}=0, \quad v_{2}=0, \quad \theta_{2}=0$, on $y=1$,

where the prime denotes derivative with respect to $y$, and $i=\sqrt{-1}$.

The analytical solutions of the zeroth-order equations are

$u_{0}(y)=-\frac{1}{6} \operatorname{Gr}(y-1) y[2+m+(m-1) y] \cos \varphi$

$\theta_{0}=1+(m-1) y$

In order to solve the first and the second-order equations (Eqs. (16) - (23)), it is appropriate to write them in a more simple form by using the notation of the stream function. The first and the second-order velocity components are written in terms of the stream functions $\bar{\psi}_{1}$ and $\bar{\psi}_{2}$ as follows:

$u_{k}=-\frac{\partial \bar{\psi}_{k}}{\partial y}$, and $v_{k}=\frac{\partial \bar{\psi}_{k}}{\partial x} \quad(k=1,2)$

Subsequently, by eliminating the pressure $p_{1}$ between Eqs. (17) And (18), and $\mathrm{p}_{2}$ between Eqs. (21) And (22), and also writing $\bar{\psi}_{1}, \theta_{1}, \bar{\psi}_{2}$ and $\theta_{2}$ as [5]

$$
\begin{aligned}
& \bar{\psi}_{1}(x, y)=e^{i L x} \psi(y), \quad \theta_{1}(x, y)=e^{i L x} t(y), \\
& \bar{\psi}_{2}(x, y)=e^{i L x} \Psi(y), \theta_{2}(x, y)=e^{i L x} \Theta(y),
\end{aligned}
$$


The first and the second-order equations reduce to the following ordinary differential equations:

$$
\begin{aligned}
& \psi^{i v}-\psi^{\prime \prime}\left[2 L^{2}+i L u_{0}\right]+\psi\left[L^{4}+i L u_{0}^{\prime \prime}+i L^{3} u_{0}\right]=G r\left(t^{\prime} \cos \varphi-i L t \sin \varphi\right) \\
& t^{\prime \prime}-t\left(L^{2}+i L u_{0} \operatorname{Pr}\right)=i L \operatorname{Pr} \psi \theta_{0}^{\prime} \\
& \Psi^{i v}-\Psi^{\prime \prime}\left[2 L^{2}+i L u_{0}\right]+\Psi\left[L^{4}+i L u_{0}^{\prime \prime}+i L^{3} u_{0}\right]+i L e^{i L x}\left(\psi^{\prime} \psi^{\prime \prime}-\psi \psi^{\prime \prime \prime}\right)=G r\left(\Theta^{\prime} \cos \varphi-i L \Theta \sin \varphi\right), \\
& \Theta^{\prime \prime}-\Theta\left(L^{2}+i L u_{0} \operatorname{Pr}\right)=i L \operatorname{Pr} \Psi \theta_{0}^{\prime}+i L \operatorname{Pr} e^{i L x}\left(\psi t^{\prime}-\psi^{\prime} t\right)
\end{aligned}
$$

Moreover, the first and the second-order boundary conditions (Eqs. (24) - (26)) are reduced to the following form:

$$
\begin{array}{lll}
\psi^{\prime}=u_{0}^{\prime}, \psi=0, t=-\theta_{0}^{\prime}, \quad \text { on } & & y=0 \\
\psi^{\prime}=0, \psi=0, t=0, \quad \text { on } & & y=1 \\
\Psi^{\prime}=u_{1}^{\prime}, \Psi=0, \Theta=-\theta_{1}^{\prime}, \text { on } & & y=0 \\
\Psi^{\prime}=0, \Psi=0, \Theta=0, & \text { on } & y=1
\end{array}
$$

Substituting the following relations

$$
\begin{aligned}
& \psi(L, y)=\sum_{j=0}^{2} L^{j} \psi_{j} \quad, \quad t(L, y)=\sum_{j=0}^{2} L^{j} t_{j}, \\
& \Psi(L, y)=\sum_{j=0}^{2} L^{j} \Psi_{j}, \quad \Theta(L, y)=\sum_{j=0}^{2} L^{j} \Theta_{j},
\end{aligned}
$$

into Eqs. (32) Through (35), and arranging the resulting equations up to the order of $L^{2}$ ( $L$ is assumed to be small, $L<<$ 1), yield the following system of the ordinary differential equations:

$$
\begin{aligned}
& \left\{\begin{array}{l}
\psi_{0}^{i v}=G r t_{0}^{\prime} \cos \varphi \\
t_{0}^{\prime \prime}=0
\end{array}\right. \\
& \left\{\begin{array}{l}
\psi_{1}^{i v}=i\left(u_{0} \psi_{0}^{\prime \prime}-u_{0}^{\prime \prime} \psi_{0}\right)+G r t_{1}^{\prime} \cos \varphi-i G r t_{0} \sin \varphi \\
t_{1}^{\prime \prime}=i \operatorname{Pr}\left(u_{0} t_{0}+\psi_{0} \theta_{0}^{\prime}\right),
\end{array}\right. \\
& \left\{\begin{array}{l}
\psi_{2}^{i v}=2 \psi_{0}^{\prime \prime}+i\left(u_{0} \psi_{1}^{\prime \prime}-u_{0}^{\prime \prime} \psi_{1}\right)+G r t_{2}^{\prime} \cos \varphi-i G r t_{1} \sin \varphi \\
t_{2}^{\prime \prime}=i \operatorname{Pr}\left(u_{0} t_{1}+\psi_{1} \theta_{0}^{\prime}\right)+t_{0}
\end{array}\right. \\
& \left\{\begin{array}{l}
\Psi_{0}^{i v}=\cos \varphi G r \Theta_{0}^{\prime} \\
\mathrm{T}_{0}^{\prime \prime}=0
\end{array}\right. \\
& \left\{\begin{array}{l}
\Psi_{1}^{i v}=i\left(u_{0} \Psi_{0}^{\prime \prime}-u_{0}^{\prime \prime} \Psi_{0}\right)+G r \Theta_{1}^{\prime} \cos \varphi-i G r \Theta_{0} \sin \varphi-i e^{i L x}\left(\psi_{0}^{\prime} \psi_{0}^{\prime \prime}-\psi_{0} \psi_{0}^{\prime \prime \prime}\right), \\
\Theta_{1}^{\prime \prime}=i \operatorname{Pr}\left(u_{0} \Theta_{0}+\Psi_{0} \theta_{0}^{\prime}\right)+i \operatorname{Pr} e^{i L x}\left(\psi_{0} t_{0}^{\prime}-\psi_{0}^{\prime} t_{0}\right),
\end{array}\right.
\end{aligned}
$$


$\left\{\begin{array}{l}\Psi_{2}^{i v}=2 \Psi_{0}^{\prime \prime}+i\left(u_{0} \Psi_{1}^{\prime \prime}-u_{0}^{\prime \prime} \Psi_{1}\right)+\operatorname{Gr}\left(\Theta_{2}^{\prime} \cos \varphi-i \Theta_{1} \sin \varphi\right)-i e^{i L x}\left(\psi_{0}^{\prime} \psi_{1}^{\prime \prime}+\psi_{0}^{\prime \prime} \psi_{1}^{\prime}-\psi_{0} \psi_{1}^{\prime \prime \prime}-\psi_{0}^{\prime \prime \prime} \psi_{1}\right), \\ \Theta_{2}^{\prime \prime}=\Theta_{0}+i \operatorname{Pr}\left(u_{0} \Theta_{1}+\Psi_{1} \theta_{0}^{\prime}\right)+i \operatorname{Pr} e^{i L x}\left(\psi_{0} t_{1}^{\prime}-\psi_{1}^{\prime} t_{0}+\psi_{1} t_{0}^{\prime}-\psi_{0}^{\prime} t_{0}\right),\end{array}\right.$

with the associated boundary conditions given by

$$
\begin{aligned}
& \left\{\begin{array}{l}
\psi_{0}^{\prime}=u_{0}^{\prime}, \psi_{0}=0, t_{0}=-\theta_{0}^{\prime}, \quad \text { at } \quad y=0 \\
\psi_{0}^{\prime}=0, \psi_{0}=0, t_{0}=0, \quad \text { at } \quad y=1,
\end{array}\right. \\
& \left\{\begin{array}{l}
\psi_{k}^{\prime}=0, \psi_{k}=0, t_{k}=0, \quad \text { at } \quad y=0 \\
\psi_{k}^{\prime}=0, \psi_{k}=0, t_{k}=0, \quad \text { at } \quad y=1, \quad(k=1,2)
\end{array}\right.
\end{aligned}
$$

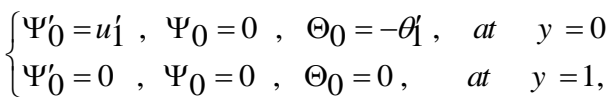

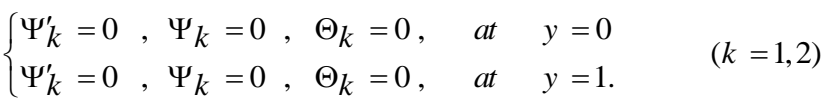

The system of the differential equations (42) - (47) together with the boundary conditions (48) - (51) are solved analytically using the MATHEMATICA code. Based on the obtained results, the solutions of the first and the second order equations are given by

$$
\begin{aligned}
& u_{1}=\operatorname{Re}\left(-\mathrm{e}^{i L x} \psi^{\prime}\right)=\psi_{i}^{\prime} \sin L x-\psi_{r}^{\prime} \cos L x \\
& v_{1}=\operatorname{Re}\left(i L \mathrm{e}^{i L x} \psi\right)=-L\left[\psi_{r} \sin L x+\psi_{i} \cos L x\right] \\
& \theta_{1}=\operatorname{Re}\left(\mathrm{e}^{i L x} t\right)=t_{r} \cos L x-t_{i} \sin L x \\
& u_{2}=\operatorname{Re}\left(-\mathrm{e}^{i L x} \Psi^{\prime}\right) \\
& v_{2}=\operatorname{Re}\left(i L \mathrm{e}^{i L x} \Psi\right) \\
& \theta_{2}=\operatorname{Re}\left(\mathrm{e}^{i L x} \Theta\right)
\end{aligned}
$$

where $\psi=\psi_{r}+i \psi_{i}$, and $t=t_{r}+i t_{i} \cdot \psi_{r}, \psi_{i}, t_{r}$ and $t_{i}$ in the above relations are given by

$$
\begin{aligned}
& \psi_{r}=\psi_{0}+L^{2} \psi_{2} \quad, \quad \psi_{i}=i L \psi_{1} \\
& t_{r}=t_{0}+L^{2} t_{2} \quad, \quad t_{i}=i L t_{1} .
\end{aligned}
$$

The non-dimensional form of the heat transfer coefficient, $h$, is written as

$$
h=-k \frac{\left(\overline{T_{l}}-\overline{T_{\infty}}\right)}{d}\left(\theta_{0}^{\prime}(y)+\operatorname{Re}\left[\varepsilon e^{i L x} t^{\prime}(y)\right]\right)
$$

The Nusselt number, $\mathrm{Nu}$, based on the width of the channel can then be obtained from

$$
N u=-\frac{h d}{\overline{T_{l}}-\overline{T_{\infty}}}=\theta_{0}^{\prime}(y)+\operatorname{Re}\left[\varepsilon e^{i L x} t^{\prime}(y)\right]
$$

The Nusselt numbers for the wavy wall $N u_{w}$, where $y=\varepsilon \cos L x$, and for the flat wall $N u_{f}$, where $y=1$, can be obtained from the following relations, respectively. 


$$
N u_{w}=\theta_{0}^{\prime}(0)+\varepsilon \operatorname{Re}\left(e^{i L x}\left[\theta_{0}^{\prime \prime}(0)+t^{\prime}(0)\right]\right),
$$

and

$$
N u_{f}=\theta_{0}^{\prime}(1)+\varepsilon \operatorname{Re}\left(e^{i L x} t^{\prime}(1)\right)
$$

\section{Validity of the solution}

For the present analytical oriented study, the theoretical work by Vajlavelu and Sastri [5] is found to be the only existing related work to this study for validation purposes. This, since no other studies have considered all the parameters or different combinations of them which are analyzed in this work.

In Fig. 2, the results of the $x$ - component of the velocity vector along the width of the vertical channel in Vajlavelu and Sastri [5] are compared with those of the present work for $L=0.01, \operatorname{Pr}=0.71$, and $L x=\pi / 2$ for some different temperature ratios $(m)$. Since the work by Vajlavelu and Sastri [5] is performed for only vertical channel $(\varphi=0)$, and a fixed amplitude, the comparison is made by setting the appropriate parameters equal between the two studies. As it can be seen in Fig. 2, very good agreements exist for the two results.

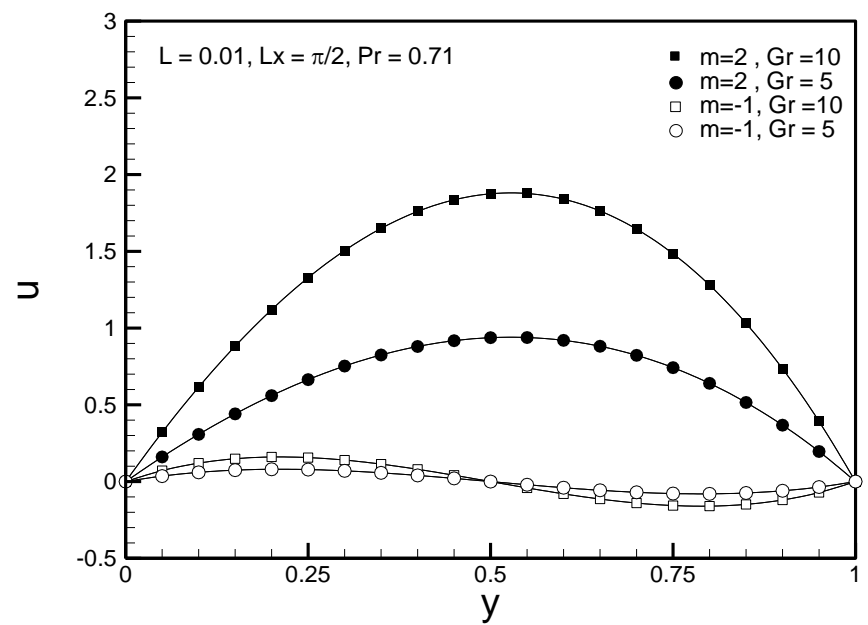

Fig. 2: Comparison of Present Study and Vajravelu and Sastri [5] Results for $x$ - Component of Velocity Vector Along a Vertical Channel Width for $L$ $=0.01, \operatorname{Pr}=0.71$, and $L x=\pi / 2$, Solidlines: Present Work; Symbols: Vajravelu and Sastri [5]

\section{Results and discussions}

Using the analytical solution, a parametric study is conducted and the effects of the pertinent parameters such as the channel angle, the amplitude, the wave length of the wall, the temperature scale $(\mathrm{m})$, and the Rayleigh number on the fluid flow and heat transfer in the fully-developed region of the channel are investigated. The results of this work are based on $\operatorname{Pr}=0.71$. However, for fluids with the Prandtl number greater than unity, the criterion of keeping $\left(R a_{d}\right)^{1 / 4}<$ $2(H / d){ }^{1 / 4}$ should hold for the fully-developed region [16] (where $R a_{d}$ is the Rayleigh number based on the wall-to-wall spacing $d$, and $H$ is the channel length), therefore, for large Rayleigh numbers, the channel height $H$ should be long enough in order to have a fully-developed region. Hence, there is a practical upper bound on the $R a$.

As far as the order of the considered wavy wall amplitude, $\varepsilon$, based on the analyzed results, it is determined that for the expansion of the field variables, up to the first power of $\varepsilon$ is quite adequate to obtain accurate solution for the flow and temperature distributions. Therefore, the results presented here are based on the linear expansions (Eq. (13)).

Figure 3 shows the streamlines and the isotherms in the fully-developed region of the channel for $L=0.01, \varphi=0^{\circ}$ (a vertical channel), $R a=10^{3}, m=2$, for different values of $\varepsilon$. As it can be seen from this figure, the streamlines follow the pattern of the wavy wall, and become parallel to the vertical direction by approaching the flat wall. The parallel isotherms show that the heat transfer in that region is basically through conduction. A closer look of Fig. 3-b indicates that the isotherms converge near the crests and diverge close to the troughs of the wavy wall. This phenomenon is more pronounced for $\varepsilon=0.1$ (Fig. 3). 
(a)
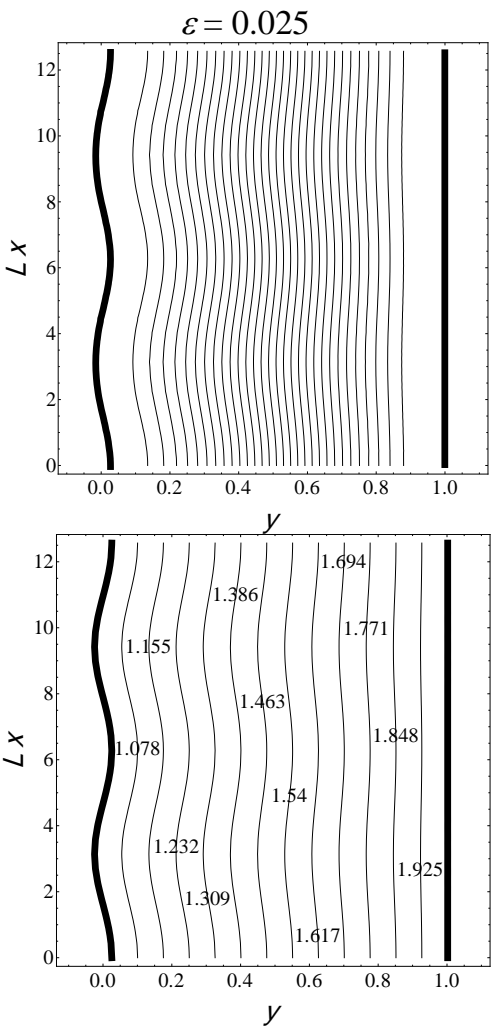
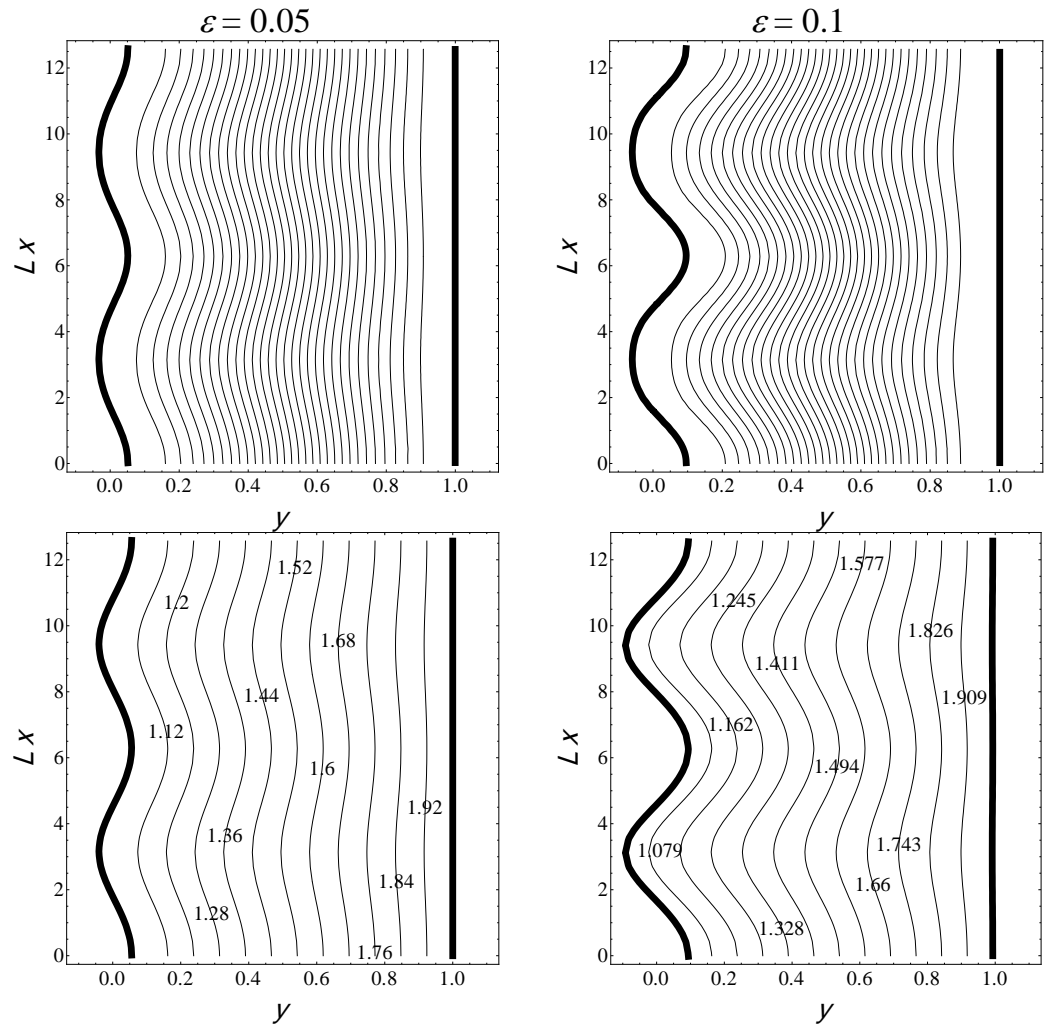

Fig. 3: Streamlines and Isotherms for the Fully-Developed Channel Flow for $L=0.01, \varphi=0^{\circ}, m=2$, and $R a=10^{3}$ for Different Values of $\varepsilon$ : (a) Streamlines; (b) Isotherms

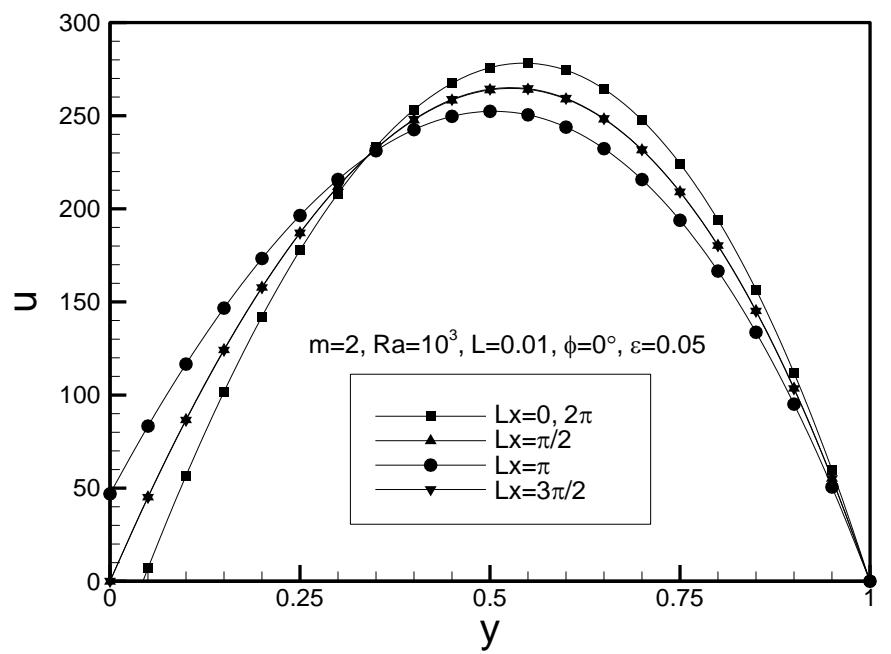

Fig. 4: The $x$-Component of Velocity Vector Along Channel Width for $L=0.01, m=2, R a=103$, and $\varepsilon=0.05$ for Different Values of $L x$

Figure 4 shows the $x$-component of the velocity vector along the channel width, for $L=0.01, \varphi=0^{\circ}, m=2, R a=10^{3}$, and $\varepsilon=0.05$ for different locations on the wavy wall, $L x$. The results are presented for four different positions along a period of the wavy wall. As the figure shows, the magnitude of the maximum velocity as well as its position along the channel width changes by moving along the channel axis. Considering a period of the wavy wall, the highest and the lowest magnitudes of the maximum velocity occur at $L x=0,2 \pi$ and $L x=\pi$, respectively. As the figure shows, when $L x$ is varied from 0 to $\pi$ (crest to valley), the maximum $x$-component velocity decreases and gets closer to the wavy wall (to the left of the channel width mid-point). A totally reverse trend, however, takes place for the maximum velocity when $L x$ varies from $\pi$ to $2 \pi$ (valley to crest)

The variations of the $x$-component of the velocity vector along the channel width for different channel inclination angles for $L=0.01, m=2, R a=10^{3}, \varepsilon=0.05$, and $L x=\pi / 2$ are depicted in Fig. 5. The buoyancy term in the momentum equation, which is the driving force for the natural convection, decreases with increasing the inclination angle $\varphi$. This results in the reduction of the $x$-component velocity vector as it can be observed from Fig. 5 . 


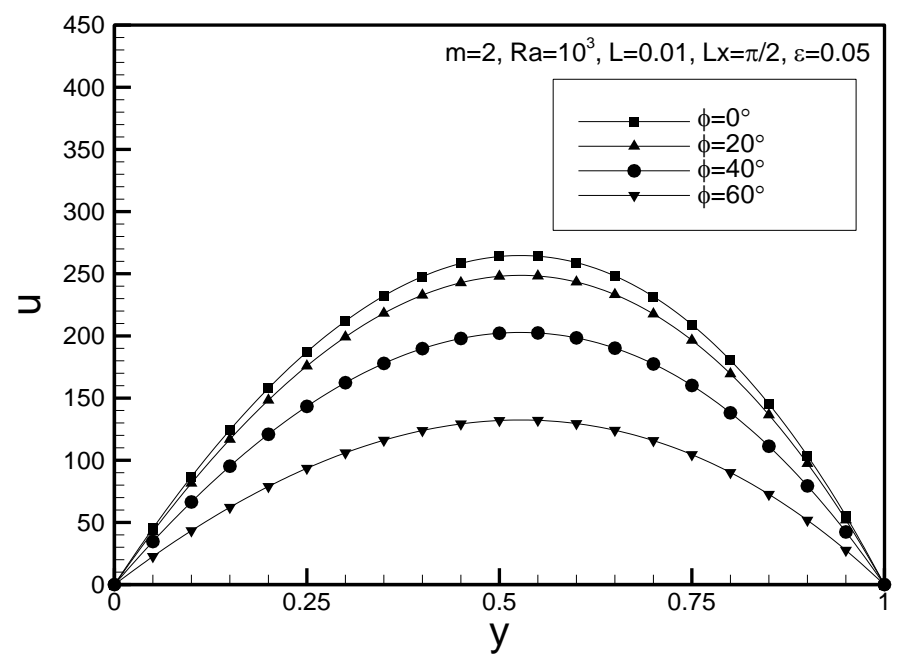

Fig. 5: Effects of Inclination Angle on $x$-Component of Velocity Vector Along Channel Width for $L=0.01, m=2, R a=10^{3}$, and $\varepsilon=0.05$

Figure 6 shows the variation of the $x$-component of the velocity vector along the channel width for different Rayleigh numbers for $L=0.01, \varphi=0^{\circ}, m=2, \varepsilon=0.05$, and $L x=\pi / 2$. As the figure shows, a substantial increase in the $x$ component of the velocity vector occurs with increasing the Rayleigh number from $10^{3}$ to $10^{4}$.

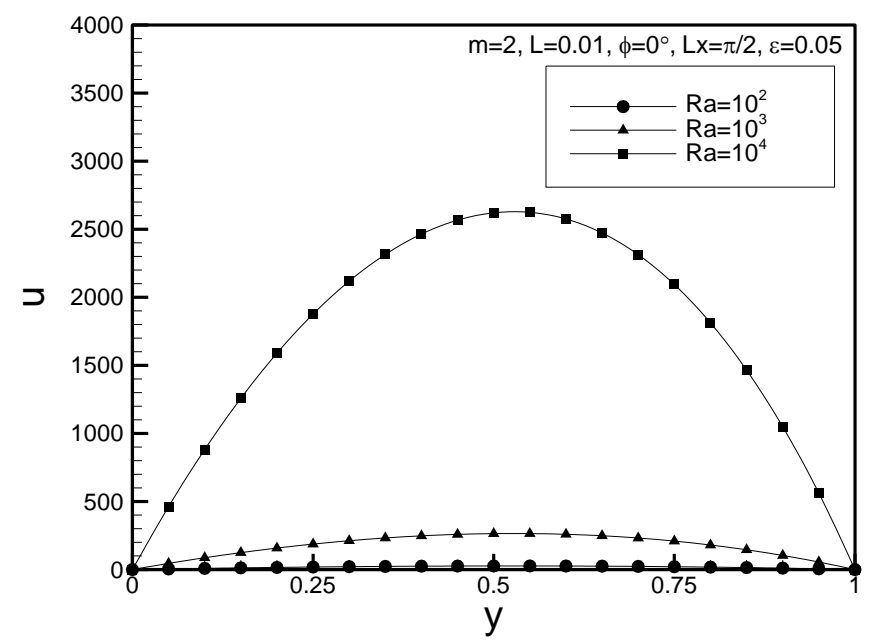

Fig. 6: Effects of The Rayleigh Number on $x$-Component of Velocity Vector Along Channel Width for $L=0.01, \varphi=0^{\circ}, m=2$, and $L x=\pi / 2$

The streamlines and isotherms for $L=0.01, \varphi=0^{\circ}, R a=10^{3}, \varepsilon=0.05$, and different values of $\mathrm{m}$ are depicted in Fig. 7 . When the temperatures of both of the walls are higher than the ambient temperature, $\overline{T_{\infty}}$ i.e. $m>0$, there is a buoyancydriven flow in the positive $x$-direction. However, for $m<0$, the temperature of one of the walls is higher, and the temperature of the other wall is lower than the ambient temperature. Under such circumstances, two distinct regions are distinguished for the fluid flow in the channel. In the area close to the wall with the higher temperature, the fluid moves upwards; while, the reverse trend is observed in the vicinity of the wall with the lower temperature (Fig. 7). This reverse trend for the flow for $m<0$ can also be observed in Fig. 8 which shows the variation of the $x$ - component of the velocity vector along the channel width for different $m$. Moreover, as Fig. 8 shows, the $x$ - component velocity increases with increasing the parameter $m$ showing that the natural convection is intensifying for higher values of $m$.

Figure 9 shows the variation of the $y$-component velocity vector along the channel width for different temperature scale parameter, $m$. The $y$-component of the velocity vector is, in general, much smaller than the $x$-component of the velocity for the vertical channel. As the figure shows, this velocity component is negative throughout the channel indicating that the fluid flows towards the wavy wall. Moreover, comparing Fig. 8 with Fig. 9 shows that the parameter $m$ has similar effects on the $x$ - and $y$-components of the velocity vector, i.e., both of the absolute values of the velocity components increase with increasing $m$. Furthermore, it is seen from Fig. 9 that the maximum absolute value of the $y$-component of the velocity vector occurs somewhere between the midsection of the channel width and the wavy wall for all values of $m$. 
(a)
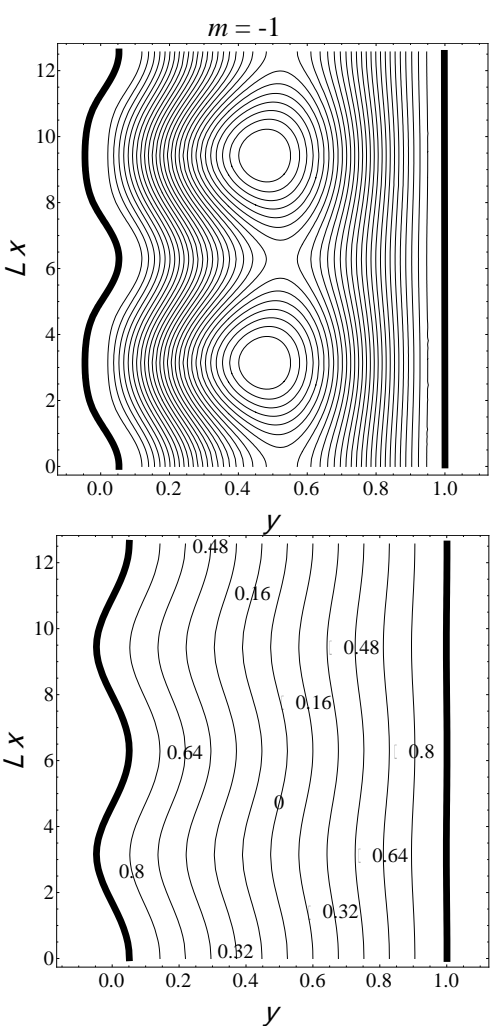
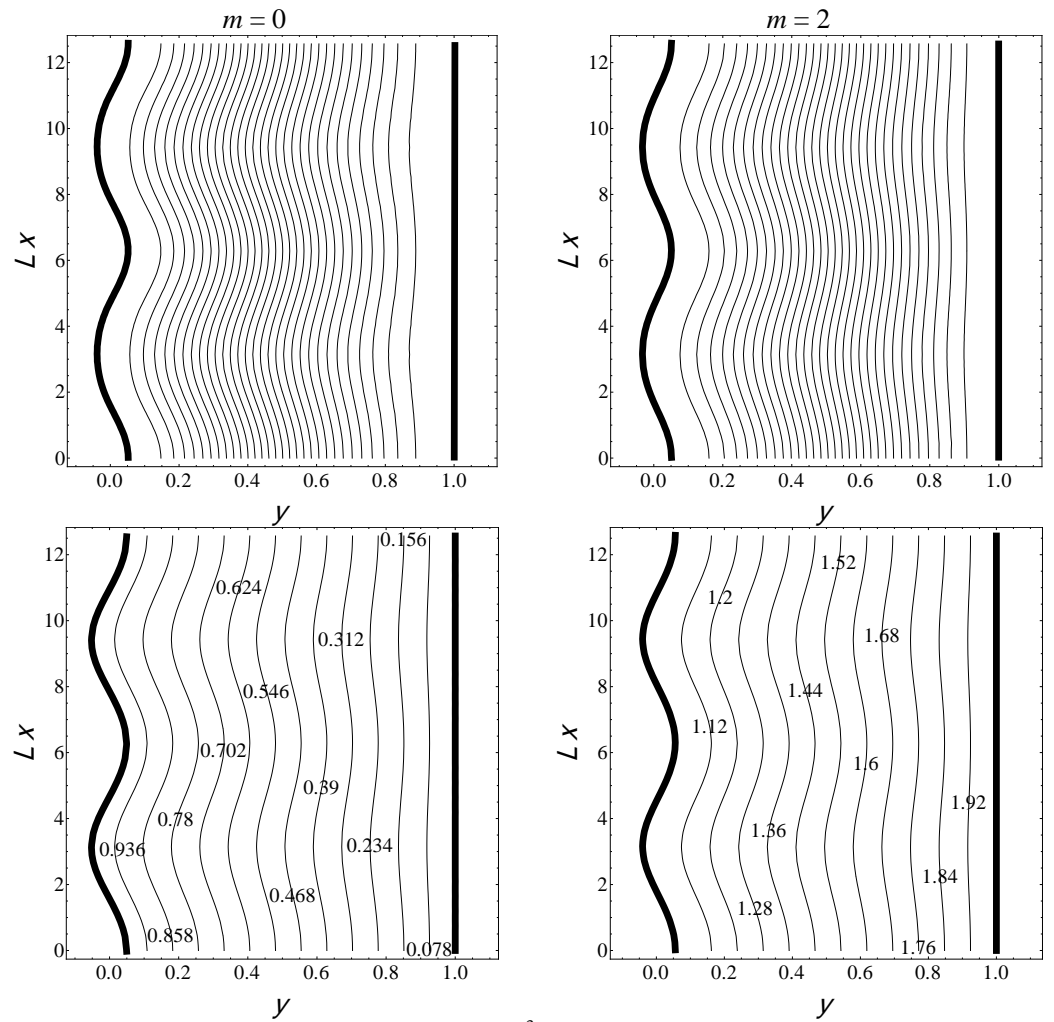

Fig. 7: Streamlines and Isotherms for Fully Developed Channel Flow for $L=0.01, \varphi=0^{\circ}, R a=10^{3}$, and $\varepsilon=0.05$, for Different Values of $m$; (a) Streamlines (b) Isotherms

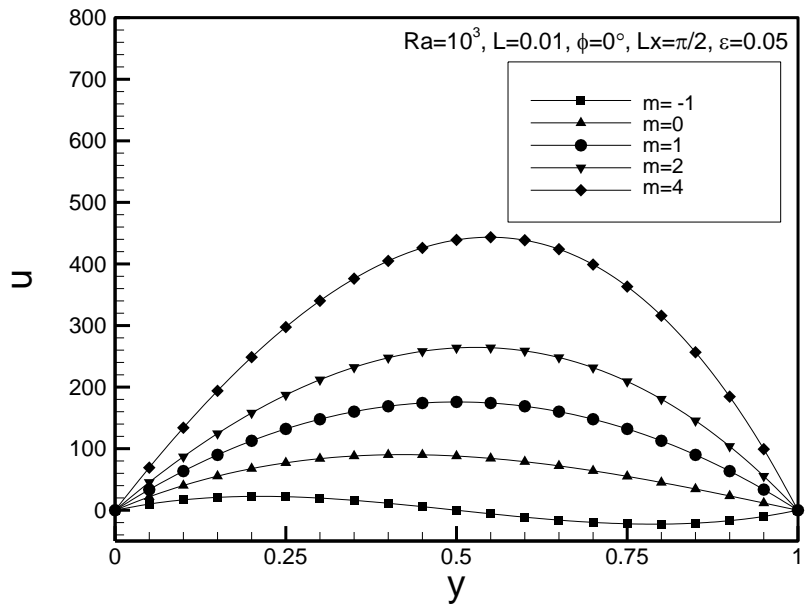

Fig. 8: Variations of $x$-Component of Velocity Vector Along Channel Width for Different $m$ For $L=0.01, \varphi=0^{\circ}, R a=10^{3}, \varepsilon=0.05$, and $L x=$ $\pi / 2$

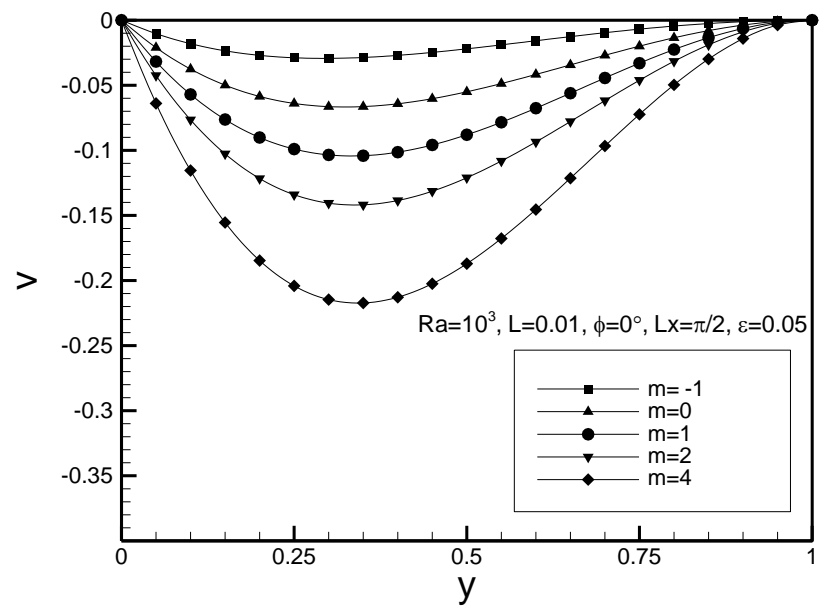

Fig. 9: Variations of $y$-Component of Velocity Vector Along Channel Width for Different $m$ For $L=0.01, \varphi=0^{\circ}, R a=10^{3}, \varepsilon=0.05$, and $L x=$ $\pi / 2$

Figure 10 magnifies the streamlines for $L=0.01, R a=10^{3}, m=2, \varepsilon=0.1$, for different values of the inclination angle, $\varphi$. From this figure, it is observed that eddies are formed within the valley of the wavy wall. This is due to the blockage of the fluid flow which corresponds to the opposite pressure gradient from the wall. It can also be observed that the absolute values of the eddies streamfunctions decrease by increasing the channel inclination angle. This is because, as the $\varphi$ increases, the strength of the buoyancy forces decrease. 

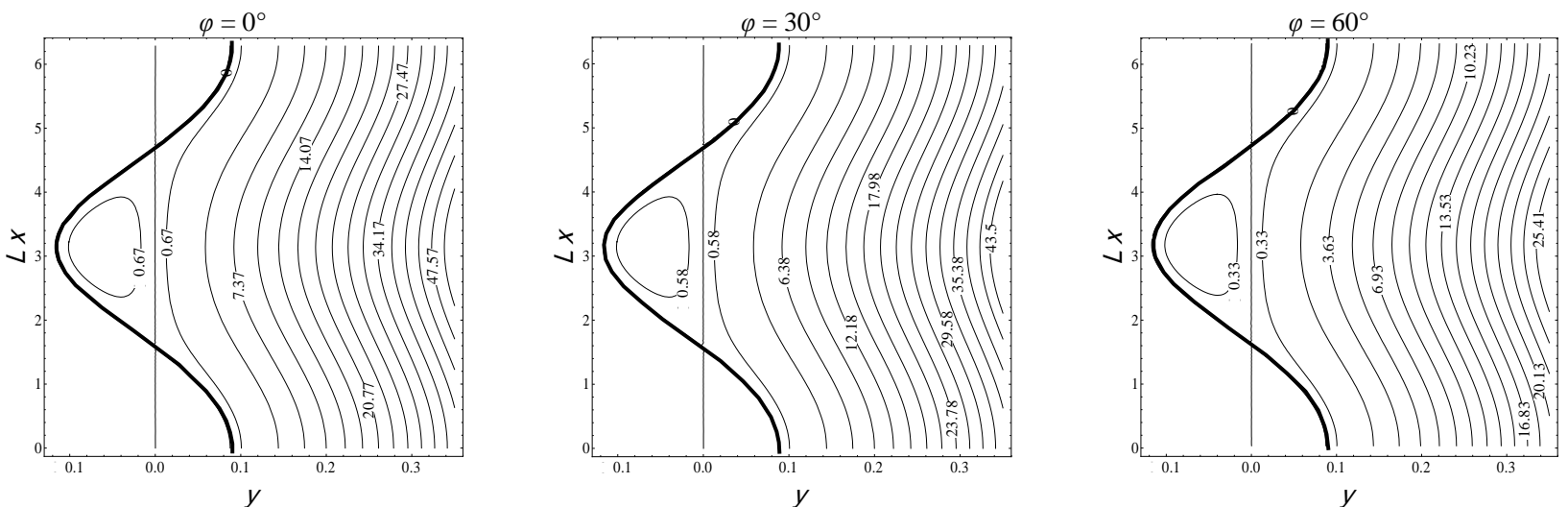

Fig. 10: Inclination Angle Effects on Channel Streamlines for $L=0.01, \varepsilon=0.1, R a=10^{3}$, and $m=2$

The effect of the scale temperature parameter, $m$, on the formation of eddies for $L=0.01, R a=10^{3}, \varphi=0^{\circ}$, and $\varepsilon=0.1$ is presented in Fig. 11. It can be seen in this figure that as $m$ increases, the strength of the eddies intensifies which shows a better transfer of heat taking place there.

The effect of the amplitude of the wavy wall, $\varepsilon$, on the flow pattern for $L=0.01, R a=10^{3}, m=2$, and $\varphi=0^{\circ}$ is depicted in Fig. 12. It can be seen that by decreasing the values of $\varepsilon$, eddies are diminished, and eventually disappear as $\varepsilon$ tends to zero.
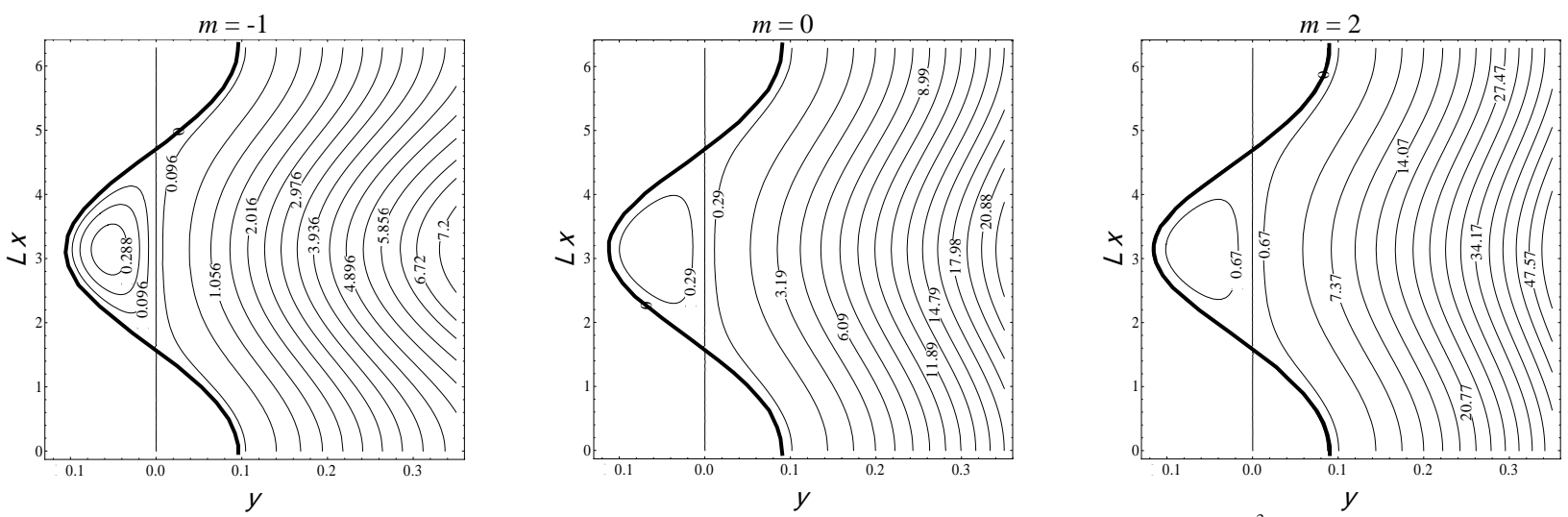

Fig. 11: Effects of Temperature Scale Variation on Channel Streamlines for $L=0.01, \varphi=0^{\circ}, R a=10^{3}$, and $\varepsilon=0.1$
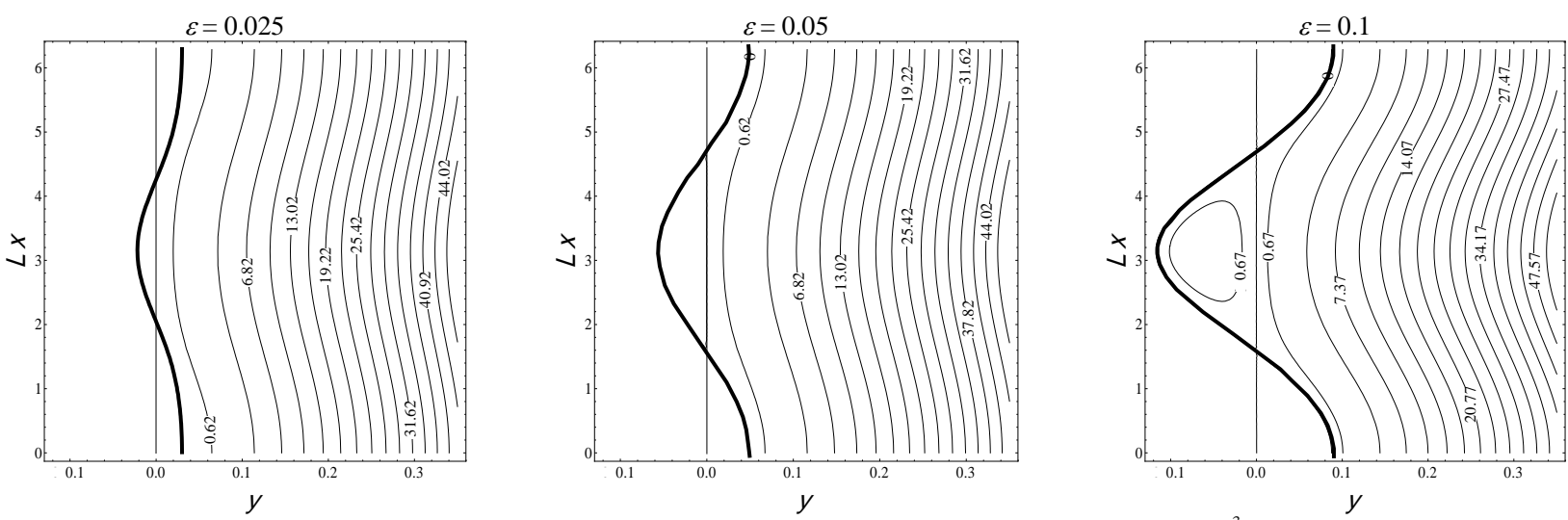

Fig. 12: Effects of Wavy Wall Amplitude on Channel Streamlines for $L=0.01, \varphi=0^{\circ}, \mathrm{Ra}=10^{3}$, and $m=2$

Figure 13 shows the variations of the local Nusselt numbers along a period of the wavy wall for $L=0.01, R a=10^{3}, m=$ $2, \varepsilon=0.05$, for different values of the inclination angle, $\varphi$. As the figure shows, the local maxima and the local minima of the Nusselt numbers occur at the crests and the troughs of the wavy wall, respectively. This is due to the fact that based on the earlier discussions; the isotherms converge near the crests and diverge close to the troughs. The figure also shows that as the inclination angle increases, the natural convection weakens and the difference between the local maximum and the local minimum of the Nusselt number decreases (Fig. 13). 


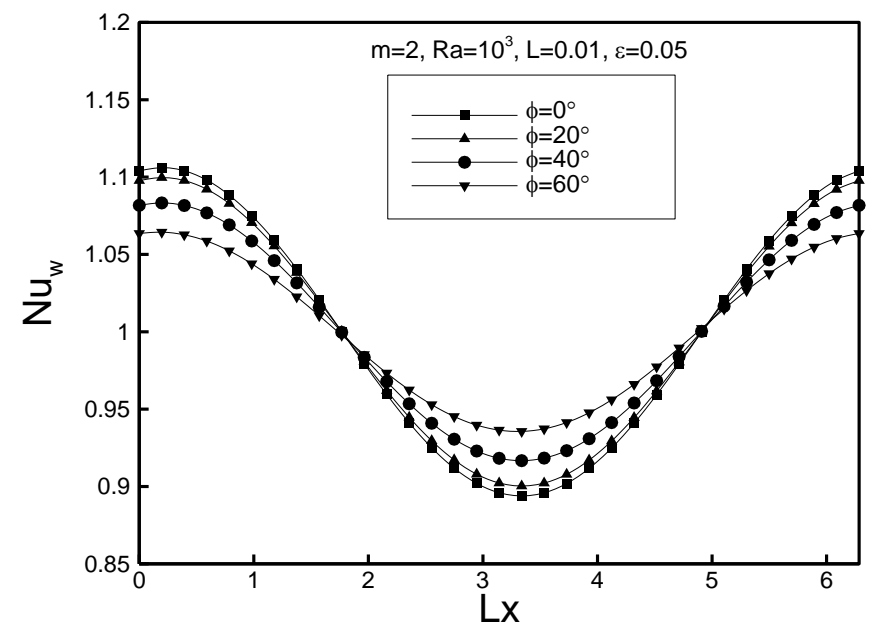

Fig. 13: Effects of Inclination Angle of Channel on the Local Nusselt Number of Wavy Wall, $L=0.01, m=2, R a=10^{3}$, and $\varepsilon=0.05$ For Different Inclination Angles

The variations of the local Nusselt number along a period of the wavy wall for different Rayleigh numbers for $L=0.01$, $\varphi=0^{\circ}, m=2$, and $\varepsilon=0.05$ is depicted in Fig. 14. As the figure shows, with increasing the Rayleigh number, the natural convection intensifies. Therefore, the local maximum of the Nusselt number profile increases at the wavy wall crests. The Rayleigh number increase, however, decreases the local Nusselt number minimum of the profile at the troughs. This, again due to the isotherms convergence and divergence near the crests and troughs of the wavy wall, respectively.

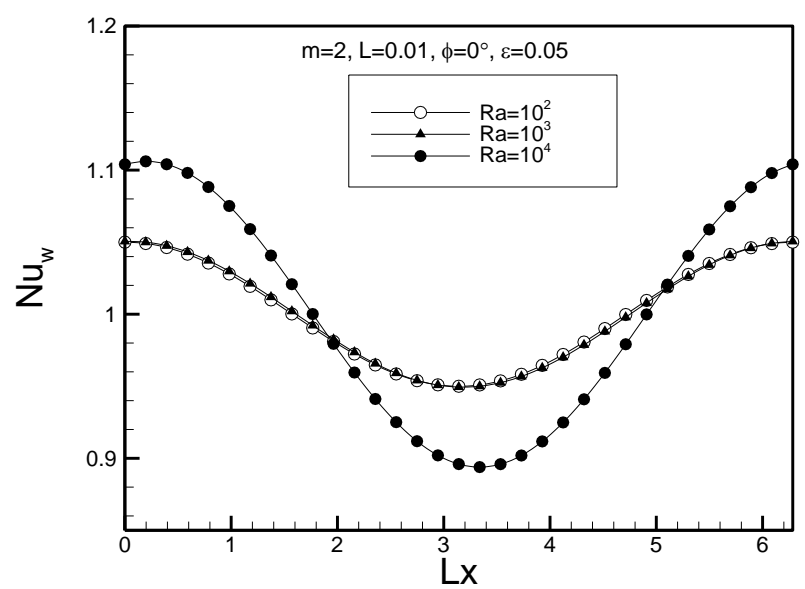

Fig. 14: Variations of the Local Nusselt Number of Wavy Wall with Respect to $L x$ For $L=0.01, m=2, \varphi=0^{\circ}$, and $\varepsilon=0.05$ for Different Rayleigh Numbers

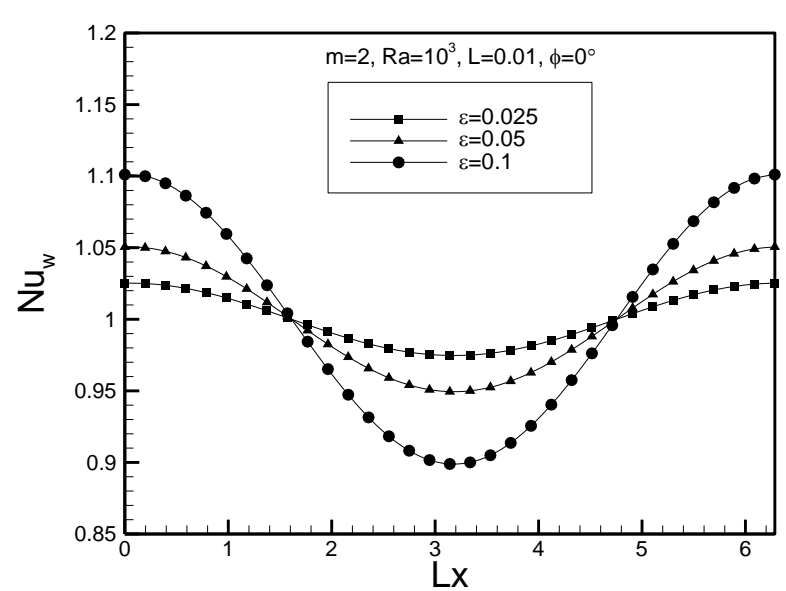

Fig. 15: Variations of the Local Nusselt Number of Wavy Wall with Respect To $L x$ for $L=0.01, \varphi=0^{\circ}, R a=10^{3}$, and $m=2$ for Different Amplitudes

The variations of the local Nusselt number of the wavy wall with respect to $L x$ for $L=0.01, m=2, R a=10^{3}, \varphi=0^{\circ}$, for different values of $\varepsilon$ are presented in Fig. 15. It is observed from this figure that the Nusselt number at the crests of the wavy wall increases by increasing the wall amplitude due to the isotherms becoming more compact at these points. The figure also shows a decrease of the local Nusselt number at the troughs as the amplitude increases.

\section{Conclusions}

The effects of a number of combinations of parameters, particularly, different combinations of the Rayleigh number, inclination angle, walls temperature-difference, and amplitude of a wavy-walled channel on the streamlines and temperature distributions for the fully developed fluid flow within the channel have been investigated. The laminar incompressible fluid flow was assumed to undergo a buoyancy-driven heat transfer due to the differential temperature difference between the two side walls of the inclined channel.

The perturbation technique based on the small amplitude of the undulated wall was utilized to solve the appropriate Navier-Stokes and energy governing equations yielding the velocity and temperature distributions for the fully developed fluid flow within the channel. Based on the obtained results, the strength of eddies formed intensifies as the natural transfer of heat enhances. The eddies, however, show to diminish as the amplitude of the undulation reduces. The illustrative results show that the difference between the local maxima and minima of the Nusselt number profiles decreases with increasing the inclination angle. 
As far as the effects of the Rayleigh number on the fluid flow velocity, the results demonstrate that the fluid flow velocity along the channel axis increases with increasing the Rayleigh number.

The performed parametric study reveals that for negative values of the wall temperature ratio, $m$, the flow in the channel, in the region close to the wall having the lower temperature, reverses its direction.

It should be noted that, although the presence of the wavy wall in this study has made it possible for the regular perturbation technique to be applicable, the method, however, shows its superiority over the conventional numerical methods, for it does not require complicated grid generation (because of the existing irregular boundaries), consumes less computing time, and invites much higher accuracy.

\section{References}

[1] Lekoudis, S. G., Nayfeh, A. H., \& Saric, W. S. (1976). Compressible boundary layers over wavy walls. Physics of Fluids, 19, 514-519. http://dx.doi.org/10.1063/1.861507.

[2] Shankar, P. N., \& Sinha, U. N. (1976). The Rayleigh problem for a wavy wall. Journal of Fluid Mechanics, 77, $243-256$. http://dx.doi.org/10.1017/S0022112076002097.

[3] Lessen, M., \& Gangwani, S. T. (1976). Effects of small amplitude wall waviness upon the stability of the laminar boundary layer. Physics of Fluids, 19, 510-513. http://dx.doi.org/10.1063/1.861515.

[4] Rees, D. A. S., \& Pop, I. (1994). Free convection induced by a horizontal wavy surface in a porous medium. Fluid Dynamics Research, 14, 151-66. http://dx.doi.org/10.1016/0169-5983(94)90026-4.

[5] Vajravelu, K., \& Sastri, K. S. (1978). Free convective heat transfer in a viscous incompressible fluid confined between a long vertical wavy wall and a parallel flat wall. Journal of Fluid Mechanics, 86, 365-383. http://dx.doi.org/10.1017/S0022112078001172.

[6] Vajravelu, K. (1980). Fluid flow and heat transfer in horizontal wavy channels. Acta Mechanica, 35, $245-258$. http://dx.doi.org/10.1007/BF01190400.

[7] Das, U. N., \& Ahmed, N. (1992). Free convective MHD flow and heat transfer in a viscous incompressible fluid confined between a long vertical wavy wall and a parallel flat wall. Indian Journal of Pure and Applied Mathematics, 23, 295-204.

[8] Das, U. N., \& Deka, R. (1992). Free convection in a viscous incompressible fluid confined between a long vertical wavy wall and a parallel flat wall: A numerical approach. Journal of Assam Science Society, 34(4), 33-43

[9] Patidar, R. P., \& Purohit G. N. (1998). Free convection flow of a viscous incompressible fluid in a porous medium between two long vertical wavy walls. Indian Journal of Mathematics, 40, 76-86.

[10] Rao, D. R. V. P., Krishna, D. V., \& Sivaprasad, R. (1987). MHD convection flow in a vertical wavy channel with temperature-dependent heat sources. Proceedings of the Indian National Science Academy, 53, 63-74.

[11] Vajravelu, K. (1989). Combined free and forced convection in hydro magnetic flows in vertical wavy channels with traveling thermal waves. International Journal of Engineering Science, 27(3), 289-300. http://dx.doi.org/10.1016/0020-7225(89)90117-1.

[12] Choudhury, R., \& Das, A. (2000). Free convection flow of a non-newtonian fluid in a vertical channel. Defence Science Journal, 50(1), $37-$ 44. http://dx.doi.org/10.14429/dsi.50.3322.

[13] Rajeev, T., \& Jain, N. C. (2004). MHD flow with slip effects and temperature-dependent heat source in a viscous incompressible fluid confined between a long vertical wavy wall and a parallel flat wall. Defence Science Journal, 54(1), 21-29. http://dx.doi.org/10.14429/dsj.54.2018.

[14] Guria, M., \& Jana, R. N. (2006). Hydrodynamic flows through vertical wavy channel with travelling thermal waves embedded in porous medium. International Journal of Applied Mechanics and Engineering, 11(3), 609-621.

[15] Muthuraj, R., \& Srinivas, S. (2010). Mixed convective heat and mass transfer in a vertical wavy channel with traveling thermal waves and porous medium. Computers \& Mathematics with Applications, 59, 3516-3528. http://dx.doi.org/10.1016/j.camwa.2010.03.045.

[16] Umavathi, J. C., \& Shekar, M. (2011). Mixed convection flow and heat transfer in a vertical wavy channel containing porous and fluid layer with traveling thermal waves. International Journal of Engineering, Science and Technology, 197(3), 196-219.

[17] Gireesha B. J., \& Mahanthesh, B. (2013). Perturbation solution for radiating viscoelastic fluid flow and heat transfer with convective boundary condition in nonuniform channel with hall current and chemical reaction. ISRN Thermodynamics, Article ID 935481, 14. http://dx.doi.org/10.1155/2013/935481.

[18] Kumar, J. P., \& Umavathi, J. C. (2013). Free convective flow in an open-ended vertical porous wavy channel with a perfectly conductive thin baffle. Heat Transfer -Asian Research, DOI: 10.1002/htj.21118. http://dx.doi.org/10.1002/htj.21118.

[19] Umavathi, J. C., \& Shekar, M. (2014). Mixed convective flow of immiscible fluids in a vertical corrugated channel with traveling thermal waves. Journal of King Saud University - Engineering Sciences, 26, 49-68. http://dx.doi.org/10.1016/j.jksues.2012.11.002.

[20] Bejan, A. (2004). Convective heat transfer. (3rd Ed.). New York: Wiley, (140-142). 